\title{
El montañismo en Patagonia-norte: una aproximación antropológica sobre sus implicancias locales y globales
}

\author{
Mountaineering in Northern Patagonia: \\ An anthropological approach to its local and global implications
}

\section{Gustavo Cayun Pichunlef*}

\footnotetext{
* Mapuche. Doctorando en Antropología por la Universidad de Buenos Aires, máster en Antropología por la Universidad Autónoma de Barcelona, licenciado en Ciencias Antropológicas por la Universidad Nacional de Río Negro. Profesor de Educación Física por la Universidad Nacional del Comahue.

-gustavocayunpichun-

lef@gmail.com

http://orcid.org/0000-0002-

6985-2688
}

RECIBIDO: 31.8.2021

ACEPTAD0: 17.11.2021

\section{Resumen}

Este trabajo visibiliza algunas de las disputas en torno a la construcción discursivo-territorial que el montañismo recrea en un enclave geográfico específico, el lago Nahuel Huapi en Patagonia (Argentina). También se analizan los modos en que las relaciones de poder y las desigualdades se imbrican en este proceso de configuración de historias, subjetividades e identidades en una región con población mapuche, preexistente a la organización estatal vigente. La hipótesis infiere que las prácticas de montañismo reproducen una historia - la oficial- de una parte de la población, mientras omite e invisibiliza los conocimientos, saberes e historias de las culturas y poblaciones subalternas involucradas, como la población mapuche. Al mismo tiempo, se recrea como una actividad inocua e inofensiva detrás de una actividad productiva de carácter global, el turismo. Cuestiono mi hipótesis analizando los procesos de construcción discursiva del territorio a partir del montañismo y reconstruyendo algunas de las relaciones y los procesos que operan por la instalación del montañismo como práctica ligada al turismo en una dinámica que articula local y global.

Palabras clave: deporte, globalización, Argentina. 


\section{Abstract}

This work makes visible some of the disputes around the discursive-territorial construction that mountaineering recreates in a specific geographical enclave, Lake Nahuel Huapi in Patagonia (Argentina). It also analyzes the ways in which power relations and inequalities are intertwined in this process of shaping histories, subjectivities and identities in a region with a Mapuche population, pre-existing to the current state organization. The hypothesis infers that mountaineering practices reproduce an official history of only a part of the population, while omitting and making invisible the knowledge, wisdom and histories of the subordinate cultures and populations involved, such as the Mapuche ethnicity. At the same time, it is recreated as an innocuous and harmless activity behind the global industry of tourism. The territorial discursive construction processes derived from mountaineering are analyzed. Relationships and operating processes due to the installation of mountaineering as a practice linked to tourism are reconstructed, in a dynamic that articulates local and global.

Keywords: sport, globalization, Argentina.

\section{Introducción}

El presente artículo recupera una investigación más amplia que llevo adelante en el marco del Doctorado en Antropología sobre las prácticas de montañismo en la región del lago Nahuel Huapi, donde se asientan la ciudad de San Carlos de Bariloche (Bariloche, en líneas siguientes) y el parque nacional Nahuel Huapi. Este enclave geográfico es reconocido nacional e internacionalmente como uno de los destinos por excelencia para el turismo invernal y estival, donde las actividades recreativas y deportivas en la naturaleza son la atracción central de los visitantes.

Considerando que el montañismo es un concepto polisémico y que, como tal, está atravesado por diversidad de prácticas, sentidos y significaciones en función de quien lo enuncia, es fundamental aclarar a qué me estoy refiriendo con esa noción. Entiendo el montañismo como una práctica corporal deportiva, enmarcada en un complejo entramado de discursos, historias, relaciones, instituciones e identidades que aún no han sido objeto de análisis antropológico en la ciudad de Bariloche. En términos deportivos, parto de considerar al deporte como:

una forma construida por la sociedad, emergente de un conjunto de manifestaciones culturales sostenidas en la dimensión de lo lúdico que, implicando un compromiso corporal regulado de forma específica en razón de su vínculo con la competencia, da lugar a la producción de formas corporales específicas fundadas en el 
orden de lo privado y que se exponen en el espacio público, convirtiéndolas en cosa pública. (Ron, 2015, p. 125)

Particularmente, en el recorte geográfico que analizo, el montañismo engloba una serie de prácticas que tienen íntima vinculación con el esquí y el «alpinismo». En simultáneo, esas prácticas son objeto de diferentes políticas públicas interjurisdiccionales, así como objeto de la formación docente y técnica tanto en una universidad pública nacional como en distintas instituciones privadas.

En este trabajo se abordan esas prácticas como un fenómeno sociocultural, político y económico que nos permite leer y comprender un entramado multiescalar (Lins Ribeiro, 2007) más amplio, que condensa múltiples actores y discursos de orden local y global. Mediante la articulación de estas dimensiones, muestro cómo el montañismo organiza una parte considerable de la vida de las personas. En ese marco, el Estado juega un papel destacado, tanto en la edificación de la belleza turística del espacio/paisaje como en la delimitación de los sujetos ideales de la práctica, con lo que genera la exclusión más o menos explícita de amplios sectores de la población y margina así otras historias, experiencias, corporalidades, comunidades y profesionales de las prácticas de montañismo. Desde esta perspectiva, el análisis propuesto se consolida en un prisma que permite descomponer, entre otras cuestiones, las construcciones sociohistóricas discursivas y la producción de subjetividades de practicantes y trabajadores vinculados al montañismo que sedimentan la hegemonía de una compleja trama que reproduce distinciones y desigualdades en un espacio concreto. Este panorama que describo ha sido estudiado desde perspectivas que no parten del montañismo, con resultados que suelen exponer de un modo compartimentado o fragmentado el entramado que pretendo analizar.

Así, en un primer momento mapeo algunos de los trabajos que intersecan las temáticas que intento analizar en un escenario más amplio que el lago Nahuel Huapi. En segundo lugar, problematizo la construcción discursiva y territorial en la que el montañismo se inserta. Luego, en un tercer momento, pongo en tensión el montañismo en términos de local/global. Por último, planteo una serie de interrogantes y procesos inteligibles al fenómeno sociocultural en cuestión.

\section{Las investigaciones del montañismo en las ciencias sociales}

Si ampliamos el lente en términos geográficos pero lo ajustamos en términos disciplinares, observamos un déficit de trabajos que problematicen las prácticas deportivas de montañismo. En ese sentido, este campo se configura en un área de vacancia en 
la que es necesario indagar a partir de sistematizar experiencias etnográficas que permitan profundizar en sus implicancias y efectos. A su vez, este panorama disciplinar también se caracteriza por el complejo entramado institucional e interdisciplinar en el que se investiga sobre la montaña en distintas regiones del mundo, en el cual se encuentran institutos especializados en un abordaje multidisciplinar con fines diversos (Moscoso Sánchez y González Fernández, 2004). A esto se suma un creciente número de adeptos que están volcándose a estas prácticas, para las que el territorio de Patagonia es un lugar privilegiado, situación que las ha ido reconfigurando con nuevas categorías y problematizándolas como fenómeno (Águila Soto, 2007).

La antropología ya venía observando esta ebullición a partir de un abordaje focalizado en lo discursivo (Feixa, 1995), un trabajo desde la perspectiva geertziana en el Himalaya (Ortner, 1999) y, más recientemente, un estudio socioantropológico con énfasis en la necesidad de establecer criterios comunes dada la diversidad de prácticas que se implementan (Moscoso, 2003). Profundizando en el caso español ${ }^{1}$ encontramos $^{2}$ investigaciones más recientes que, por ejemplo, abordan el excursionismo como desagregación del montañismo puntualizando en el proceso histórico de la práctica (Roma i Casanovas, 2010), el surgimiento de esta en la burguesía barcelonesa (Andreu et al., 1995), los desafíos que implica pensar el montañismo en la actualidad (Nasarre Sarmiento, 2016), dimensionar las actividades en el medio natural (Queixalós et al., 2016) y el recurrente interés por clasificar las prácticas de montañismo (Olivera Betrán y Olivera Betrán, 2016).

Por otro lado, vemos una incipiente institucionalización de lo que se denomina antropología de la montaña, en la que empiezan a sucederse espacios de encuentro - enmarcados en jornadas - con énfasis en los aportes que la antropología y la arqueología realizan en contextos y ecosistemas de montaña, como es el caso de las jornadas organizadas por la Escuela Nacional de Historia y Antropología de México. Aquí resulta importante destacar que el abordaje que se observa en este espacio se encuentra hegemonizado por investigaciones con foco en las culturas indígenas y poblaciones prehispánicas, situación que evidencia una fuerte presencia de la arqueología como disciplina vectora de los espacios.

Inda y Rosaldo (2002) identifican que la antropología ofrece una atención concreta a la agencia humana, a las prácticas de la vida cotidiana por las que los sujetos

1 Retomo este país, y particularmente el caso catalán, debido a que en el período 2017-2018 realicé una estancia en ese territorio para cursar el Máster en Antropología en la Universidad Autónoma de Barcelona, cuyo trabajo final se orientó a la comparación de dos prácticas de montañismo situadas: el excursionismo y el senderismo. Cabe aclarar que en este artículo no problematizo las tensiones relativas a circunscribir el caso catalán al Estado español, pero es innegable que en el contexto del 2017-2018 en Cataluña se experimentó una muy notoria efervescencia y una conflictividad con la idea de pertenecer a España. 
median en los procesos de globalización. Desde este enfoque, un abordaje en torno al montañismo nos lleva a preguntarnos cómo y cuáles son los procesos a gran escala (o flujos de sujetos y objetos) mediante los cuales el mundo se vuelve cada vez más interconectado y de qué maneras los sujetos responden a este proceso en formas culturales específicas.

Ajustando la búsqueda al enclave del Nahuel Huapi, sobre la línea de investigación de prácticas corporales vinculadas al montañismo en la región de Patagonia Norte, encontramos un trabajo historiográfico que se enfoca en el rol que tuvieron en la construcción identitaria nacional las prácticas corporales e instituciones como el scoutismo, Parques Nacionales y el Club Andino Bariloche (Chiocconi et al., 2011). También hallamos un trabajo que aborda la relación entre el esquí y la política estatal hacia mediados del siglo XX (Chiocconi, 2015).

Por otra parte, las investigaciones vinculadas a la formación de profesores de Educación Física con orientación al montañismo son escasas en Argentina, incluso en el Centro Regional Universitario Bariloche de la Universidad Nacional del Comahue ${ }^{2}$ (CRUBUNComa), única institución de Sudamérica que forma a profesores con las incumbencias profesionales que les permiten habilitarse como guías de trekking e instructores de esquí. Excepcionalmente, encontramos una compilación con foco en las prácticas de andinismo en la Educación Física del CRUB-UNComa (Goicoechea Gaona y López, 2016), con un énfasis descriptivo y técnico de las prácticas que se realizan puntualmente en dos asignaturas del profesorado: Deportes Regionales Estivales I (tercer año) y Deportes Regionales Estivales II (cuarto y último año de la carrera). Por otro lado, encontramos dos tesis de posgrado presentadas para la obtención de una maestría en la Universidad Nacional de Quilmes (Palacio, 2017; Mársico, 2019), que, con diferentes perspectivas, abordan la relación entre la enseñanza de diversas prácticas de montaña y sus dimensiones pedagógica y política.

\section{El montañismo como fenómeno sociocultural desde el Nawel Wapi mapu}

¿Por qué Nawel Wapi mapu? En esta nominación problematizo una de las tesis mencionadas inicialmente, según la cual algunas historias han quedado relegadas frente

Esta situación de vacancia en parte se explica por el bajo número de profesionales docentes que puedan dirigir proyectos de investigación, situación que ha llevado a cierto padrinazgo institucional en el que inicialmente cumplió este rol un sector de docentes del Profesorado en Educación Física de la Universidad Nacional de La Plata. Este escenario, en ocasiones, ha generado tensiones dentro del CRUB-UNComa, por la distancia geográfica y temática que tienen los profesionales con las prácticas de montañismo. 
a la historia que opera como «oficial» en el urbanocentrado relato nacional. Puntualmente, la noción más extendida del territorio que intento describir es la de Patagonia, nombre creado desde afuera, por visitantes expedicionarios, clérigos y militares. Como construcción, refiere a lo salvaje, lo grotesco, a una nominación des-ligada y des-territorializada de los sentidos que los pueblos le otorgan a su vida, a partir de una caracterización colonial de las poblaciones preexistentes a estas «expediciones» y «visitas fugaces» (como es el caso del pueblo mapuche, entre otros) y a una recreación territorial más reciente- de un espacio «desértico», metáfora de lo inhabitado por la «civilización»y, por lo tanto, «conquistable», adjudicable y privatizable; es decir, territorio sujeto a adquirirse como propiedad privada.

No obstante, la Patagonia experimentó históricamente un rápido pasaje que la llevó de ser considerada un lugar inhóspito a ser uno de los epicentros del turismo en Latinoamérica. Claudia Hammerschmidt (2016) señala que tanto la historia de su poblamiento como la construcción discursiva de su espacio fue realizada por «descubridores» llegados desde afuera, que la han fundado y proyectado entre la idealización paradisíaca y lo infernal, inventando un «espacio imaginario» (Livon-Grosman, 2003) correspondiente a sus ansias materiales e ideológicas. Estas han sido sobre todo producciones europeas y de las metrópolis chilenas y argentinas que se sirvieron del sur para construirse e identificarse con el norte.

Frente a esto, recupero perspectivas historiográficas y territoriales que reconstruyen la historia desde el pueblo mapuche, la cual ha sido excluida y silenciada por los relatos hegemónicos (Moyano, 2013) y que, aún hoy, sigue estando expuesta en un contexto de creciente estigmatización y criminalización de este pueblo (Escolar et al., 2010). En este sentido, es vital posicionarse en una mirada que problematice los grandes relatos -en este caso, de Bariloche-, los cuales pueden clasificarse en cuatro grandes grupos. En primer lugar, aquellos que giran en torno a la narrativa nostálgica, institucional y fotográfica de Ricardo Vallmitjana (1989). En segundo lugar, aquellos que confluyen en la construcción arquitectónica «fundacional» de Bariloche con una mirada estadocéntrica y conservadora, una de cuyas narrativas por excelencia es la de Exequiel Bustillo (1968-1999). En tercer lugar, íntimamente vinculado a los dos primeros, el relato ligado a la metáfora de Bariloche como la Suiza argentina (Kropff, 2005; Navarro Floria, 2008). Por último, los estudios que profundizan en los documentos, cartas y biografías de eclesiásticos, entre los que se destacan trabajos que mapean las intervenciones de misioneros (Nicoletti, 2014).

En otra línea historiográfica, menos oficial y periférica, que parte del postulado de que el montañismo, en sentido amplio, implica acciones de desplazamiento en terrenos que presentan características geomorfológicas de montaña, pareciera vislumbrarse que en el Nawel Wapi mapu (una de las formas mapuche de denominar este territorio como 'isla del tigre') esta historia de desplazamientos no comienza con las «hazañas de los 
migrantes europeos» ni con «los pioneros». Al contrario, diversos registros muestran que la región cuenta con asentamientos previos de comunidades que transitaron el territorio de mar a mar (Moyano, 2016), que existen prácticas de desplazamiento de larga data - como el arreo de ganado o la trashumancia-y, aún más, el trabajo arqueológico en torno a las canoas monóxilas (Braicovich y Caracotche, 2008; Lezcano et al., 2015) da cuenta de una navegación milenaria en el vasto Nawel Wapi lafken (la última palabra significa 'lago' en mapuzugun). Por lo tanto, el tránsito de poblaciones autodefinidas a partir de un territorio cordillerano identificado como waizuf mapu ('cordillera' en mapuzugun) y con principal fuerza en su gran lago es, por lo menos, milenario.

De este modo, el relato historiográfico vigente en los espacios académicos, en las prácticas de montañismo y en el sentido común de la población desconoce — por omisión y por eurocentrismo- las prácticas de los pueblos preexistentes a la constitución de los Estado-nación-territorio (Delrio, 2010). En consecuencia, la relación del hombre y su ambiente en clave de montañismo, parece originarse - y limitarse - a la acotada y des-situada experiencia europea que ubica a la primera ascensión al Mont Blanc - tomada como hito fundacional del alpinismo - como el evento en el que el hombre comienza a relacionarse de un modo diferente con la montaña (Puga y Palacio, 2011). De igual forma, los hitos o acontecimientos que marcaron la historia del montañismo remiten a una concepción occidental de conquista de las cumbres asociada a ideas de exigencia y sacrificio tales como ascender más alto, en condiciones meteorológicas adversas, de forma autosuficiente e individual, rompiendo límites, antecedentes y, en ello, marcando récords.

Lo anterior también ha «otorgado» a los representantes del montañismo de plataforma europea la facultad de asignar nuevos nombres a montañas, ríos, lagos, valles y filos por encima de los usos, saberes, interpretaciones y formas de nominar de los pueblos preexistentes. El caso del territorio vinculado al Nawel Wapi lafken y el Parque Nacional que lo contiene es un ejemplo ilustrativo de este accionar. Así, en contraste con la nominación mapuche, en la que el gen ('ser') de la montaña, cerro, valle, lago, río tiene protagonismo a partir de sus características y en función de una ontología que le otorga mongen ('vida' y 'entidad'), en la experiencia del montañismo la nominación está librada al gusto de los conquistadores. En esta línea, Claudia Briones (2019) viene señalando que, en el marco de los desacuerdos que atraviesan nuestras sociedades contemporáneas, el pueblo mapuche-tewelche ${ }^{3}$ marca disensos más complejos que los ideológicos. zación del Nawel Wapi mapu o cercanas, que reivindican su identidad en términos de los dos pueblos preexistentes a la constitución del Estado-nación argentino, el pueblo mapuche y el pueblo tewelche. Al mismo tiempo, esta autoidentificación da cuenta de la interacción pasada y presente de estos pueblos, la cual no solo puede verse en la construcción identitaria sino, también, en el empleo de un lenguaje en el que confluyen palabras de ambas lenguas, mapuzungun y gününa yajik. 
En este punto, las demandas de pueblos indígenas de la región del Nawel Wapi van mostrando bordes ontológicos y epistemológicos también comprometidos en el mapa de desacuerdos (Briones, 2019).

En el montañismo se producen y recrean un conjunto de prácticas deportivas y recreativas modernas que surgen en un contexto europeo, proliferan a escala global y se insertan en distintos territorios locales, donde adquieren diferentes sentidos y significados. Al mismo tiempo, este conjunto se constituye por una diversidad de actividades que en el Nawel Wapi mapu se han institucionalizado como trekking o senderismo, escalada en roca, escalada en hielo, esquí alpino, esquí nórdico, esquí de travesía, rapel, tirolesa, canopy, canoning. Este recorte excluye algunas actividades que, de algún modo, están vinculadas al montañismo en la experiencia del Nawel Wapi, tales como el kayak de travesía, la escalada libre o en boulders naturales, el parapente, entre otras. El relevamiento anterior surge de definir el montañismo en el sentido amplio previamente señalado, que conlleva delimitarlo como conjunto de prácticas desarrolladas en espacios que presentan geoformas en las que predominan montañas, valles y cerros.

Es importante destacar que el montañismo se recrea en Patagonia a partir de su homología con el alpinismo europeo, aunque aquí, pensado respecto a su formación geológica más extensa - la cordillera de los Andes-, se denomina andinismo. Así, el andinismo se forja a partir de las prácticas de los pioneros (migrantes europeos de principios del siglo XX), invisibilizando y omitiendo las milenarias prácticas previas de cuya existencia dan cuenta numerosos registros. Lo anterior se articula con el turismo, práctica que, para el caso del Nawel Wapi mapu, fue impulsada por el Estado argentino junto con la creación del parque nacional Nahuel Huapi en la década del treinta (Navarro Floria, 2008).

\section{¿Cómo se insertan las prácticas de montañismo en un escenario global? ¿Qué lugares ocupa Patagonia?}

El clásico y precursor trabajo de Sherry Ortner (1999) muestra la tensión entre los modos de practicar montañismo de dos mundos inconmensurables: el de sherpas y el de sahibs. La autora reconstruye cómo las comunidades locales, en este caso de las poblaciones cercanas al monte Everest, los Himalayas, tienen concepciones diferentes en torno a las implicancias y los efectos de ascender a la montaña y los motivos para ello -que para los sherpas nepalíes se constituye en una forma económica de subsistir-. Ortner (1999) nos muestra que hay vida más allá del discurso, aun del más totalizador, 
y hay modos de reacomodar el mundo más allá de los marcos discursivos de los escaladores y montañistas, que en definitiva son los clientes. Para los sherpas (etnia nepalí conocida en el ámbito global del montañismo por su vinculación al porteo de equipo y alimentos para una expedición), el ascender pone en juego otras cuestiones, tales como la ofensa a los dioses; más aún, deben conducir a los sahibs (modo de designar en su lengua a los montañistas) lo más cercanamente posible de sus propias inquietudes. De esta forma, y entendiendo que la globalización implica que no todas las personas ni todos los lugares participan por igual en los circuitos que interconectan el globo (Inda y Rosaldo, 2002), podemos problematizar el lugar que ocupan ciertas prácticas que se consolidan como industrias globales en contextos y territorios que presentan marcadas diferencias culturales con sus «practicantes».

Con este punto de partida, puede entenderse que el montañismo se ha transformado en una práctica cultural que trasciende las fronteras de los Estados-nación. Tanto es así que las noticias en torno a las hazañas que se logran en un punto del globo por actores particulares, individualizados y socialmente situados alimentan la construcción transnacional de paisajes imaginarios (Appadurai, 2002). En sus inicios, esta práctica implicó un interés por conocer aquello que se presentaba como inaccesible, «virgen» o inhóspito, para luego dar paso a un marcado interés por alcanzar las grandes cumbres. Países como Italia, Polonia, Francia, Austria, Alemania, Estados Unidos o el Reino Unido se disputaban los primeros ascensos en el escenario territorial europeo (Moscoso, 2002). La difusión de la actividad y un marcado nacionalismo que buscaba plantar bandera en regiones cada vez más inhóspitas y lejanas fueron consolidando la idea de la montaña como escenario a conquistar.

Su difusión, a partir de la creación de clubes, configuró un panorama que permite ver cómo circulan flujos culturales en algunos de los paisajes que identifica Appadurai (2002). Marcadamente en términos étnicos se observa la circulación de turistas «montañistas» y de una mano de obra técnica — guías - que trabaja en ambos hemisferios según la temporada para la que está certificada. ${ }^{4} \mathrm{~A}$ su vez, en términos mediáticos se construye un paisaje de imágenes que recrean redes de interconexión de sitios en un escenario global amplio, que va desde las altas cumbres del Himalaya, con escalas en los Alpes y los Pirineos, pasa por grandes parques nacionales de Norteamérica hasta llegar

Esto también muestra que se establecen cánones de certificación mundial, a través de la Unión Internacional de Asociaciones de Guías de Montaña, donde podemos encontrar guías de alta montaña que trabajan en temporadas invernal y estival indistintamente en cualquier parte del mundo, y guías de montaña y trekking que trabajan generalmente en temporada estival o como ayudantes de guías de alta montaña. 
a la cordillera sudamericana..$^{5}$ Este flujo de trabajadores, expediciones y paquetes turísticos que circulan entre continentes deja ver que la globalización es, también, un proceso sumamente desigual. Por ejemplo, mientras que algunas personas poseen los recursos políticos y económicos para recorrer el mundo (como montañistas con sponsors), muchas otras tienen poco o ningún acceso al transporte, ${ }^{6}$ a los medios de comunicación, a un boleto de avión o incluso a una llamada telefónica.

En este escenario, se consolida un mercado de avances tecnológicos que permite manejar un vocabulario común entre los practicantes de la actividad, independientemente del lugar en el que se encuentren. Se crea así un conglomerado de marcas líderes alrededor del globo - tales como The North Face, Mountain Hardwear, Patagonia, Salomon, Columbia - que, además de producir equipamiento, adquieren reconocimiento y publicidad mediante el auspicio de grandes eventos de montaña ${ }^{7}$ en todo el mundo. Inda y Rosaldo (2002) también lo observan entre las características de la globalización, puesto que, desde ropa, alimentos y música hasta arquitectura, cine y televisión, no se puede negar que ciertos estilos y marcas —occidentales - alcanzan prominencia mundial, a tal punto que se pueden encontrar prácticamente en cualquier parte del planeta. A esto se suma que ser «practicante» constituye una clave para entender lo que Appadurai (2002) identifica como el pasaje de un fetichismo de la mercancía a los fetichismos de la producción y del consumidor.

El primero, el de la producción, refiere a la ilusión creada por los sitios donde tiene lugar la producción transnacional contemporánea. Aquí las relaciones de producción experimentan una transnacionalidad sin precedentes. Para el caso del montañismo, las marcas líderes no pierden su referencia de origen nacional, pero establecen sus fábricas en lugares donde pueden abaratar los costos de producción (generalmente en países asiáticos), como lo ejemplifica la vinculación de la marca The North Face con Estados Unidos. De este modo, la localidad se torna un fetiche que encubre y desfigura las fuerzas dispersas por todo el globo, que, en realidad, son las que dinamizan la producción. Aquí la alienación se da en el marco de una dinámica espacial que es crecientemente global.

El segundo, el fetichismo del consumidor, implica que este fue transformado, se lo impulsa a pensar y a creer que es un actor — runner, trekker, ochomilista, entre otros-

En este sentido se identifican tres grandes referencias: la cordillera Blanca en Bolivia y Perú, el Aconcagua (montaña más alta de América) y la Patagonia (compuesta por gran cantidad de atractivos para el alpinismo clásico, tales como Frey, Cochamó, Chaltén, Torres del Paine).

6 El trabajo de Ortner (1999) muestra cómo para los sherpas el trabajar en la montaña, además de un rédito económico y una mejor paga frente a las posibilidades del lugar, también les ha permitido conocer otros lugares del mundo a partir de invitaciones de sus clientes/empleadores.

7 Un ejemplo de megaevento de montaña, con varias ediciones, puede encontrarse en https://elcrucecolumbia.com/. 
, un protagonista, cuando en el mejor de los casos es alguien que simplemente escoge en un mercado de consumo. Lo anterior nos permite pensar al montañismo también como plataforma para un mercado cada vez más isomórfico. Con esto quiero señalar que se evidencia un doble proceso en el que, por un lado, se acerca a un público cada vez más amplio, ofreciendo lo último en tecnología y equipamiento (con cadenas como Decathlon, Vértic o Barrabés para el caso europeo), situación que puede considerarse positiva en el sentido de que hace más accesibles prácticas que requieren un equipamiento costoso. Al mismo tiempo, este proceso — que me atrevo a designar como decathlonización - contiene una instancia performativa en la que se subjetivan los modos en que las actividades deben practicarse. Con esto me refiero a que los «practicantes» son moldeados para tener y vestir un equipo con determinadas características, entre las que lo fluorescente, la liviandad cada vez mayor y una especificidad de cada producto para determinadas acciones se exacerban y se consolidan como indispensables. Peor aún, se generan objetivos que llevan a diseminar las ideas y concepciones de cómo vivir esos espacios de montaña, en las que la forma cada vez más extendida es hacer cumbre, llegar lo más rápido posible y generar un registro audiovisual del hecho.

\section{Conclusión}

Lo planteado en este trabajo de aproximación permite identificar algunas tensiones que pueden explicitarse en forma de interrogante: ¿qué pasa cuando las prácticas que se extienden transgreden normas, reglas o mandatos y omiten historias y prácticas de los lugares a los que llegan? ¿De qué formas pueden contener el avance global los Estados y sus políticas públicas en aras de fortalecer las identidades e historias locales? ¿Qué procesos son inteligibles a partir de analizar el montañismo como práctica sociocultural?

En este apartado me ocuparé solo del último interrogante, quizás el más ameno, para hilvanar alguna lectura de forma arriesgada. Puedo decir que el montañismo excede a la deportivización, en términos de análisis, y da lugar a entender procesos vinculados a la turistificación, la individualización, la subjetivación de la montaña, la tecnificación, lo que denomino como decathlonización y la despolitización de estas prácticas deportivas.

Entendiendo que el turismo, actividad íntimamente ligada al montañismo, implica un complejo de relaciones entre grupos humanos mediante actividades que impactan sobre los territorios, la turistificación implica la masificación de estas relaciones y su impacto sobre el medio ambiente, las ciudades, los tejidos sociales y las economías locales, entre otros. 
Por deportivización entiendo el proceso mediante el cual el espacio en que se realizan las actividades - la montaña - se concibe exclusivamente en relación con un fenómeno social y cultural que reproduce las lógicas del deporte (competencia, institucionalización, calendarios, megaeventos, entre otras). A su vez, este proceso instala toda una serie de equipos y modos «correctos» — traducidos en técnicas de movimientoque estandarizan y estereotipan la forma de transitar y vivir el espacio, lo que devendría en una tecnificación.

El proceso de subjetivación, en tanto, lo acoto al desarrollo de gustos, intereses y modos de relación con la montaña. Este último proceso, muchas veces, se lleva a cabo desde perspectivas, miradas y enfoques despolitizados, en los que las relaciones de poder y las desigualdades sociales son relegadas en los análisis o bien ocupan lugares periféricos.

En esa línea, entiendo que la incorporación de una lectura crítica que dé cuenta de los procesos señalados permite complejizar nuestro entendimiento de la práctica sociocultural del montañismo. Consecuentemente, el análisis realizado nos invita a trascender su instalación como una actividad inocua e inofensiva, para visibilizarla también como actividad económica que, asociada al turismo y al fenómeno deportivo, genera efectos muy dispares sobre las comunidades de incidencia.

\section{Bibliografía}

Águila Soto, C. (2007). Las actividades físicas de aventura en la naturaleza ¿Un fenómeno moderno o posmoderno? Apunts: Educación Física y Deportes, 89, 81-87.

Andreu, E., Lagardera, F., y Rovira, G. (1995). El excursionismo catalán y los deportes de montaña. Apunts: Educación Física y Deportes, 41, 80-86.

Appadurai, A. (2002). Disjuncture and difference in the global cultural economy. En J. X. Inda y R. Rosaldo (eds.), The anthropology of globalization: A reader (pp. 46-64). Londres: Blackwell.

Braicovich, R., y Caracotche, S. (2008). Una biografía de las canoas monóxilas de la región andina norpatagónica: Perspectivas para su memoria y conservación. Recuperado de http://www.hechohistorico.com.ar/Trabajos/Jornadas\%20de\%20Bariloche \%20-\%202008/Braicovich-Caracotche.pdf.

Briones, C. (2019). Conflictividades interculturales: Demandas indígenas como crisis fructíferas. Bielefeld: Bielefeld University Press.

Bustillo, E. (1999). El despertar de Bariloche: Una estrategia patagónica. Buenos Aires: Sudamericana.

Chiocconi, M. (2015). La trascendencia política del esquí: Una razón de Estado en la Argentina de la primera mitad del siglo XX. En L. Méndez y A. Podlubne (comps.), 
Tiempo de jugar, tiempo de aprender: Educación, museos y prácticas corporales en la Patagonia Norte, 1910-1955 (pp. 213-240). Buenos Aires: Prometeo Libros.

Chiocconi, M., Chiappe, M., y Podlubne, A. (2011). ¡Todo por la patria! Nacionalismo, prácticas corporales y tiempo libre en asociaciones civiles: Región del Nahuel Huapi. En L. Méndez (dir.), Historias en movimiento: Cuerpo, educación y tiempo libre en la Norpatagonia 1884-1945 (pp. 181-247). Rosario: Prohistoria.

Delrio, W. (2010). Memorias de expropiación: Sometimiento e incorporación indígena en la Patagonia: 1872-1943. Bernal: Universidad Nacional de Quilmes.

Escolar, D., Delrio, W., y Malvestitti, M. (2010). Criminalización y distorsión de las demandas indígenas en Argentina: La construcción mediática del pueblo Mapuche como no-originario. Journal de la Société des Américanistes, 96(1), 293-295.

Feixa, C. (1995). La aventura imaginaria: Una visión antropológica de las actividades físicas de aventura en la naturaleza. Apunts: Educación Física y Deportes, (41), 36-43.

Goicoechea Gaona, M. V., y López, H. E. (comps.). (2016). Las prácticas de andinismo en educación física. Bariloche: Eduardo Hugo López.

Hammerschmidt, C. (ed.). (2016). Patagonia literaria: Fundaciones, invenciones y emancipaciones de un espacio geopolítico y discursivo. Potsdam: INOLAS.

Inda, X., y Rosaldo, R. (2002). Introduction: A world in motion. En J. X. Inda y R. Rosaldo (eds.), The anthropology of globalization: A Reader (pp. 1-34). Londres: Blackwell.

Kroppf, L. 2005. Bariloche: ¿una Suiza Argentina? Desde la Patagonia: Difundiendo Saberes, (2), 32-37.

Lezcano, M., Hajduk, A., y Albornoz, A. (2015). Arqueología del lago Nahuel Huapi: Modelos de uso de los ambientes y de los recursos faunísticos. Macroscopia: Divulgación Técnico-Científica del Patrimonio Natural y Cultural del Parque Nacional Nahuel Huapi, 4(4), 15-22.

Lins Ribeiro, G. (2007). Poder, redes e ideología en el campo del desarrollo. Tabula Rasa, (6), 173-193.

Livon-Grosman, E. (2003). Geografías imaginarias: El relato de viajes y la construcción del espacio patagónico. Buenos Aires: Beatriz Viterbo.

Mársico, L. (2019). Currículums en disputa: La incorporación del esquí en la propuesta curricular de las escuelas públicas de nivel primario de San Carlos de Bariloche, Río Negro (1999-2013). (Tesis de Maestría). Universidad Nacional de Quilmes, Bernal, Argentina. Recuperado de https://ridaa.unq.edu.ar/handle/20.500.11807/1906? locale-attribute=en.

Moscoso Sánchez, D., y González Fernández, M. (2004). La montaña como observatorio de los social. Córdoba (España): IESA. (IESA Working Paper Series, 21-04).

Moscoso, D. (2003). La montaña y el hombre en los albores del siglo XXI. Cuarte (Huesca): Barrabes. 
Moyano, A. (2013). Komütuam descolonizar la historia mapuche en Patagonia. Buenos Aires: Alum Mapu.

Moyano, A. (2016). A ruego de mi superior, Antonio Modesto Inakayal. Viedma: Fondo Editorial Rionegrino.

Nasarre Sarmiento, J. M. (2016). Los retos del montañismo en el siglo XXI: Respuestas normativas. Apunts: Educación Física y Deportes, 2(124), 100-107.

Navarro Floria, P. (2008). El proceso de construcción social de la región del Nahuel Huapi en la práctica simbólica y material de Exequiel Bustillo (1934-1944). Revista Pilquen: Sección Ciencias Sociales, 9(9).

Nicoletti, M. A. (2014). Pasado y presente: Los jesuitas de la misión Nahuel Huapi (16701674 y 1704-1717) y la devoción mariana. IHS: Antiguos Jesuitas en Iberoamérica, 2(1), 41-64.

Olivera Betrán, J., y Olivera Betrán, A. (2016). Las actividades físicas de aventura en la naturaleza (AFAN): Revisión de la taxonomía (1995-2015) y tablas de clasificación e identificación de las prácticas. Apunts: Educación Física y Deportes, 2(124), 71-88.

Ortner, S. (1999). Thick resistance: Death and the cultural construction of agency in Himalayan mountaineering. En S. Ortner (ed.), The fate of «Culture»: Clifford Geertz and beyond. Los Ángeles: University of California Press.

Palacio, M. (2017). El trekking y la escalada en la formación de profesores de educación física con orientación en montaña de la Universidad Nacional del Comahue: De una práctica significativa a una práctica enseñable. (Tesis de maestría). Universidad Nacional de Quilmes, Bernal, Argentina. Recuperado de http://ridaa.unq.edu.ar/ handle/20.500.11807/503.

Puga, J., y Palacio, M. (2011). Encuentros entre el andinismo y la educación física: Historia del profesorado del Centro Regional Universitario Bariloche. En E. Rozengardt y F. Acosta (comps.), Historia de la educación física y sus instituciones: Continuidades y rupturas (pp. 299-320). Buenos Aires: Miño y Dávila.

Queixalós, F., Yuba, E., y Labrador, V. (2016). Hacia un nuevo paradigma de la actividad deportiva en el medio natural. Apunts: Educación Física y Deportes, 2(124), 114-121.

Roma i Casanovas, F. (1990). E-xcursionismes 2.0: L'excursionisme català a l'inici del segle XXI: Una visió des de l'antropologia (Tesis de doctorado). Universitat Ramon Lull, Barcelona.

Ron, O. (2015). Deporte, deportes. En C. Carballo (dir.), Diccionario crítico de la educación física académica: Rastreo y análisis de los debates y tensiones del campo académico de la educación física en Argentina (pp. 119-126). Buenos Aires: Prometeo Libros.

Vallmitjana, R. (1989). Bariloche, mi pueblo. Buenos Aires: Fundación Antorchas. 